\title{
Discrete spectral synthesis
}

\author{
László Székelyhidi \\ Institute of Mathematics, University of Debrecen \\ e-mail: szekely@math.klte.hu
}

\begin{abstract}
Discrete spectral analysis and synthesis study the description of translation invariant function spaces over discrete Abelian groups. The basic building bricks are the exponential monomials. A remarkable result of R. J. Elliot in 1965 claimed that spectral synthesis holds on any Abelian group, which means that the exponential monomials span a dense linear subspace in any pointwise-closed translation invariant linear space of complex valued functions over the group. Unfortunately, the proof of this theorem had several gaps. In this paper we give a short survey about the present status of discrete spectral analysis and synthesis, we show that Elliot's theorem is false, we give a necessary condition for Abelian groups to have spectral synthesis and we formulate a conjecture about a possible characterization of Abelian groups having spectral synthesis.
\end{abstract}

Key Words: spectral synthesis, torsion free rank, polynomial functions

AMS Classification Number: 43A45, 39A70, 20K15

\section{Introduction}

Spectral analysis and spectral synthesis deal with the description of translation invariant function spaces over locally compact Abelian groups. We consider the space $\mathcal{C}(G)$ of all complex valued continuous functions on a locally compact Abelian group $G$, which is a locally convex topological linear space with respect to the pointwise linear operations (addition, multiplication with scalars) and to the topology of uniform convergence on compact sets. Continuous homomorphisms of $G$ into the additive topological group of complex numbers, and into the multiplicative topological group of nonzero complex numbers are called additive and exponential functions, respectively. A polynomial is a finite linear combination of products of additive functions and an exponential monomial is a product of a polynomial and an exponential function. Linear combinations of exponential monomials are called exponential polynomials. 
Suppose that a closed linear subspace in the space $\mathcal{C}(G)$ is given, which is translation invariant, which means that if $f$ belongs to this subspace then the translate $\tau_{y} f$ of $f$ by $y$, defined by

$$
\tau_{y} f(x)=f(x+y)
$$

belongs to the subspace as well, for any $x, y$ in $G$. Such subspaces are called varieties and these are the main objectives of spectral analysis and spectral synthesis.

It turns out that exponential functions, or more generally, exponential monomials can be considered as basic building bricks of varieties. A given variety may or may not contain any exponential function or exponential monomial of the above mentioned form. If it contains an exponential function, then we say that spectral analysis holds for the variety. An exponential function in a variety can be considered as a kind of spectral value and the set of all exponential functions in a variety is called the spectrum of the variety. It follows that spectral analysis for a variety means that the spectrum of the variety is nonempty. On the other hand, the set of all exponential monomials contained in a variety is called the spectral set of the variety. It turns out that if an exponential monomial belongs to a variety, then the exponential function appearing in the representation of this exponential monomial belongs to the variety, too. Hence if the spectral set of a variety is nonempty, then also the spectrum of the variety is nonempty and spectral analysis holds. There is, however an even stronger property of some varieties, namely, if the spectral set of the variety span a dense subspace of the variety. In this case we say that spectral synthesis holds for the variety. It follows, that for nonzero varieties spectral synthesis implies spectral analysis. If spectral analysis, resp. spectral synthesis holds for every variety on an Abelian group, then we say that spectral analysis holds, resp. spectral synthesis holds on the Abelian group. A famous and pioneer result of L. Schwartz [1] exhibits the situation by stating that if the group is the reals with the Euclidean topology, then spectral values do exist, that is, any nonzero variety contains an exponential function, the spectrum is nonempty, spectral analysis holds. Furthermore, spectral synthesis also holds in this situation: there are sufficiently many exponential monomials in the variety in the sense that their linear hull is dense in the subspace.

An interesting particular case is presented by discrete Abelian groups. Here the problem seems to be purely algebraic: all complex functions are continuous, and convergence is meant in the pointwise sense. The archetype is the additive group of integers: in this case the closed translation invariant function spaces can be characterized by systems of homogeneous linear difference equations with constant coefficients. It is known that these function spaces are spanned by exponential monomials corresponding to the characteristic values of the equation, together with their multiplicities. In this sense the classical theory of homogeneous linear difference equations with constant coefficients can be considered as spectral analysis and spectral synthesis on the additive group of integers.

The next simplest case is the case of systems of homogeneous linear difference equations with constant coefficients in several variables, or, in other words, spectral 
analysis and spectral synthesis on free Abelian groups with a finite number of generators. As in this case a structure theorem is available, namely, any group of this type is a direct product of finitely many copies of the additive group of integers, it is not very surprising to have the corresponding - nontrivial - result by M. Lefranc [2]: on finitely generated free Abelian groups spectral analysis and spectral synthesis holds for any closed translation invariant subspace.

Based on these results the natural question arises: what about other discrete Abelian groups? In his 1965 paper [4] R. J. Elliot presented a theorem on spectral synthesis for arbitrary Abelian groups. However, in 1987 Z. Gajda drew my attention to the fact that the proof of Elliot's theorem had several gaps. Since then several efforts have been made to solve the problem of discrete spectral analysis and spectral synthesis on Abelian groups. In the subsequent paragraphs we present a summary about the status of these problems. From now on we consider only discrete Abelian groups and all the above mentioned concepts are meant in the discrete setting.

\section{Spectral analysis and spectral synthesis on fini- tely generated Abelian groups}

The first general result about spectral synthesis is due to M. Lefranc on free Abelian groups of finite rank, that means, on groups of the form $\mathbb{Z}^{k}$ with some nonnegative integer $k$ (see [2]).

Theorem 2.1. Spectral synthesis holds for any free Abelian group of finite rank.

Using the following simple lemma we can clarify the connection between spectral synthesis and spectral analysis.

Lemma 2.2. Let $G$ be an Abelian group, $V$ a variety in $\mathcal{C}(G), p: G \rightarrow \mathbb{C}$ a nonzero polynomial and $m: G \rightarrow \mathbb{C}$ an exponential function. If the exponential monomial $p m$ belongs to $V$, then $m$ belongs to $V$, too.

Proof. The statement is obvious if $p$ is a nonzero constant. On the other hand, if $p$ is a nonconstant polynomial, then $\Delta_{y} p$ is a nonzero polynomial for some $y$ in $G$, with degree one less than that of $p$. Moreover, by the identity

$$
\Delta_{y} p(x) m(x)=p(x+y) m(x)-p(x) m(x)=p(x+y) m(x+y) m(-y)-p(x) m(x)
$$

which holds for each $x, y$ in $G$ it follows that the exponential monomial $\left(\Delta_{y} p\right) m$ belongs to $V$ for each $y$ in $G$. Hence our statement follows by induction on the degree of $p$.

From this lemma we infer the following theorem.

Theorem 2.3. If spectral synthesis holds for an Abelian group, then also spectral analysis holds for it. 
Using Theorem 2.1 we have the following easy consequence.

Theorem 2.4. Spectral analysis holds for any free Abelian group of finite rank.

The following theorem makes it possible to extend the above results.

Theorem 2.5. If spectral synthesis holds for an Abelian group then it holds for its homomorphic images, too.

Proof. Suppose that $G$ is an Abelian group, $H$ is a homomorphic image of $G$ and let $F: G \rightarrow H$ be a surjective homomorphism. If $V$ is a variety in $\mathcal{C}(H)$, then we let

$$
V_{F}=\{f \circ F: \quad f \in V\} .
$$

Using the surjectivity of $F$ a routine calculation shows that $V_{F}$ is a variety in $\mathcal{C}(H)$. Let $\Phi$ be an exponential monomial in $V_{F}$ of the form

$$
\Phi(x)=P\left(A_{1}(x), A_{2}(x), \ldots, A_{n}(x)\right) M(x),
$$

where $A_{1}, A_{2}, \ldots, A_{n}$ are linearly independent additive functions on $G, M$ is an exponential on $G$, and $P$ is a complex polynomial in $n$ variables. By Lemma 2.2 the exponential $M$ is in $V_{F}$, too, hence $M=m \circ F$ holds for some $m$ in $V$. If $u, v$ are arbitrary in $H$, then $u=F(x)$ and $v=F(y)$ for some $x, y$ in $G$, which implies

$$
\begin{gathered}
m(u+v)=m(F(x)+F(y))=m(F(x+y))=M(x+y)=M(x) M(y)= \\
=m(F(x)) m(F(y))=m(u) m(v) .
\end{gathered}
$$

As $m$ is never zero, hence $m$ is an exponential in $V$. On the other hand, (2.1) implies that

$$
q(x)=P\left(A_{1}(x), A_{2}(x), \ldots, A_{n}(x)\right)=p(F(x))
$$

holds for each $x$ in $G$ with some function $p: H \rightarrow \mathbb{C}$. We show that $p$ is a polynomial on $H$. Using the Newton Interpolation Formula and the Taylor Formula in several variables it follows easily that the functions $A_{1}, A_{2}, \ldots, A_{n}$ can be expressed as a linear combination of some translates of $q$. On the other hand, if $F(x)=F(y)$ for some $x, y$ in $G$, then $q(x+z)=q(y+z)$ holds for each $z$ in $G$, hence $A_{i}(x)=A_{i}(y)$ for $i=1,2, \ldots, n$. It follows that we can define the functions $a_{i}: H \rightarrow \mathbb{C}$ for $i=1,2, \ldots, n$ by the equation

$$
a_{i}(u)=A_{i}(F(x))
$$

where $x$ is arbitrary in $G$ with the property $F(x)=u$. Further, we see immediately that $a_{i}$ is additive for $i=1,2, \ldots, n$. On the other hand,

$$
p(u)=p(F(x))=P\left(A_{1}(x), A_{2}(x), \ldots, A_{n}(x)\right)=P\left(a_{1}(u), a_{2}(u), \ldots, a_{n}(u)\right)
$$

holds for any $u$ in $H$, hence $p$ is a polynomial on $H$. This means that the exponential monomial $\Phi$ above has the form $\Phi=\varphi \circ F$ with some exponential monomial $\varphi$ in $V$. Finally, it is straightforward to verify that if the exponential monomials span a dense subspace in $V_{F}$, then the corresponding exponential monomials span a dense subspace in $V$, so our proof is complete. 
Using the well-known fact that every finitely generated Abelian group is the homomorphic image of some free Abelian group of finite rank we have the following result.

Theorem 2.6. Spectral synthesis and spectral analysis holds for any finitely generated Abelian group.

At this point a simple question can be formulated: is there any non-finitely generated Abelian group, on which spectral synthesis, or spectral analysis holds?

\section{Spectral analysis and spectral synthesis on arbi- trary Abelian groups}

In 1965 R. J. Elliot published the following result in the Proc. Cambridge Phil. Soc. (see [4]):

Theorem 3.1. Spectral synthesis holds on any Abelian group.

Of course a theorem of this type would have closed all open problems concerning discrete spectral analysis and spectral synthesis. Unfortunately, in 1990 the polish mathematician Zbigniew Gajda called my attention to the fact that the proof of Elliot's theorem had several gaps. After several efforts of Gajda and myself we were unable either to fill those gaps or to find a counterexample to Elliot's result. Obviously, the question about spectral analysis on arbitrary Abelian groups turned to be open again. In this respect we could prove the following result (see [9]).

Theorem 3.2. Spectral analysis holds on every Abelian torsion group.

Proof. We show that every nonzero variety in $\mathcal{C}(G)$ contains a character. Let $V$ be any nonzero variety in $\mathcal{C}(G)$. Then by the Hahn-Banach theorem $V$ is equal to the annihilator of its annihilator, that is, there exists a set $\Lambda$ of finitely supported complex measures on $G$ such that $V$ is exactly the set of all functions in $\mathcal{C}(G)$ which are annihilated by all members of $\Lambda$ :

$$
V=V(\Lambda)=\{f \mid f \in \mathcal{C}(G),\langle\lambda, f\rangle=0 \text { for all } \lambda \in \Lambda\}
$$

We show that for any finite subset $\Gamma$ in $\Lambda$ its annihilator, $V(\Gamma)$ contains a character. Indeed, let $F_{\Gamma}$ denote the subgroup generated by the supports of the measures belonging to $\Gamma$. Then $F_{\Gamma}$ is a finitely generated torsion group. The measures belonging to $\Gamma$ can be considered as measures on $F_{\Gamma}$ and the annihilator of $\Gamma$ in $\mathcal{C}\left(F_{\Gamma}\right)$ will be denoted by $V(\Gamma)_{F_{\Gamma}}$. This is a variety in $\mathcal{C}\left(F_{\Gamma}\right)$. It is also nonzero. Indeed, if $f$ belongs to $V$ then its restriction to $F_{\Gamma}$ belongs to $V(\Gamma)_{F_{\Gamma}}$. If, in addition, we have $f\left(x_{0}\right) \neq 0$ and $y_{0}$ is in $F_{\Gamma}$, then the translate of $f$ by $x_{0}-y_{0}$ belongs to $V$, its restriction to $F_{\Gamma}$ belongs to $V(\Gamma)_{F_{\Gamma}}$ and at $y_{0}$ it takes the value $f\left(x_{0}\right) \neq 0$. Hence $V(\Gamma)_{F_{\Gamma}}$ is a nonzero variety in $\mathcal{C}\left(F_{\Gamma}\right)$. As $F_{\Gamma}$ is finitely generated, by Theorem 2.6 spectral analysis holds, and, in particular $V(\Gamma)_{F_{\Gamma}}$ contains exponential functions. 
As $F_{\Gamma}$ is a torsion group, any exponential function on $F_{\Gamma}$ is a character. That means, $V(\Gamma)_{F_{\Gamma}}$ contains a character of $F_{\Gamma}$. It is well-known (see e.g. [3]) that any character of $F_{\Gamma}$ can be extended to a character of $G$, and obviously any such extension belongs to $V(\Gamma)$.

Now we have proved that for any finite subset $\Gamma$ of the set $\Lambda$ the annihilator $V(\Gamma)$ contains a character. Let $\operatorname{char}(V)$ denote the set of all characters contained in $V$. Obviously $\operatorname{char}(V)$ is a compact subset of $\widehat{G}$, the dual of $G$, because $\operatorname{char}(V)$ is closed and $\widehat{G}$ is compact. On the other hand, the system of nonempty compact sets $\operatorname{char}(V(\Gamma))$, where $\Gamma$ is a finite subset of $\Lambda$, has the finite intersection property:

$$
\operatorname{char}\left(V\left(\Gamma_{1} \cup \Gamma_{2}\right)\right) \subseteq \operatorname{char}\left(V\left(\Gamma_{1}\right)\right) \cap \operatorname{char}\left(V\left(\Gamma_{2}\right)\right) .
$$

We infer that the intersection of this system is nonempty, and obviously

$$
\emptyset \neq \bigcap_{\Gamma \subseteq \Lambda} \operatorname{char}(V(\Gamma)) \subseteq \operatorname{char}(V) .
$$

That means, $\operatorname{char}(V)$ is nonempty, and the theorem is proved.

This theorem presents a partial answer to our previous question: as obviously there are Abelian torsion groups which are not finitely generated, hence there are non-finitely generated Abelian groups on which spectral analysis holds.

In 2001 G. Székelyhidi in [8] presented a different approach to the result of Lefranc, and he actually proved that spectral analysis holds on countably generated Abelian groups, further, his method strongly supported the conjecture that spectral analysis - hence also spectral synthesis - might fail to hold on free Abelian groups having no generating set with cardinality less than the continuum. At the 41st International Symposium on Functional Equations in 2003, Noszvaj, Hungary we presented a counterexample to Theorem 3.1 of Elliot in [4]. The counterexample depends on the following observation (see [10]).

Theorem 3.3. Let $G$ be an Abelian group. If there exists a symmetric bi-additive function $B: G \times G \rightarrow \mathbb{C}$ such that the variety $V$ generated by the quadratic function $x \mapsto B(x, x)$ is of infinite dimension, then spectral synthesis fails to hold for $V$.

Proof. Let $f(x)=B(x, x)$ for all $x$ in $G$. By the equation

$$
f(x+y)=B(x+y, x+y)=B(x, x)+2 B(x, y)+B(y, y)
$$

we see that the translation invariant subspace generated by $f$ is generated by the functions $1, f$ and all the additive functions of the form $x \mapsto B(x, y)$, where $y$ runs through $G$. Hence our assumption on $B$ is equivalent to the condition that there are infinitely many functions of the form $x \mapsto B(x, y)$ with $y$ in $G$, which are linearly independent. This also implies that there is no positive integer $n$ such that $B$ can be represented in the form

$$
B(x, y)=\sum_{k=1}^{n} a_{k}(x) b_{k}(y),
$$


where $a_{k}, b_{k}: G \rightarrow \mathbb{C}$ are additive functions $(k=1,2, \ldots, n)$. Indeed, the existence of a representation of this form would mean that the number of linearly independent additive functions of the form $x \mapsto B(x, y)$ is at most $n$.

It is clear that any translate of $f$, hence any function $g$ in $V$ satisfies

$$
\Delta_{y}^{3} g(x)=0
$$

for all $x, y$ in $G$ : this can be checked directly for $f$. Hence any exponential $m$ in $V$ satisfies the same equation, which implies

$$
m(x)(m(y)-1)^{3}=0
$$

for all $x, y$ in $G$, and this means that $m$ is identically 1 . It follows that any exponential monomial in $V$ is a polynomial. By the results in [5] (see also [6]) and by $(3.2) g$ can be uniquely represented in the following form:

$$
g(x)=A(x, x)+c(x)+d
$$

for all $x$ in $G$, where $A: G \times G \rightarrow \mathbb{C}$ is a symmetric bi-additive function, $c: G \rightarrow \mathbb{C}$ is additive and $d$ is a complex number. Here "uniqueness" means that the "monomial terms" $x \mapsto A(x, x), x \mapsto c(x)$ and $d$ are uniquely determined (see [6]). In particular, any polynomial $p$ in $V$ has a similar representation, which means that it can be written in the form

$$
p(x)=\sum_{k=1}^{n} \sum_{l=1}^{m} c_{k l} a_{k}(x) b_{l}(x)+c(x)+d=p_{2}(x)+c(x)+d
$$

with some positive integers $n, m$, additive functions $a_{k}, b_{l}, c: G \rightarrow \mathbb{C}$ and constants $c_{k l}, d$. Suppose that $p_{2}$ is not identically zero. By assumption, $p$ is the pointwise limit of a net formed by linear combinations of translates of $f$, that means, by functions of the form (3.1). Linear combinations of functions of the form (3.1) can be written as

$$
\varphi(x)=c B(x, x)+A(x)+D,
$$

with some additive function $A: G \rightarrow \mathbb{C}$ and constants $c, D$. Any net formed by these functions has the form

$$
\varphi_{\gamma}(x)=c_{\gamma} B(x, x)+A_{\gamma}(x)+D_{\gamma}
$$

By pointwise convergence

$$
\lim _{\gamma} \frac{1}{2} \Delta_{y}^{2} \varphi_{\gamma}(x)=\frac{1}{2} \Delta_{y}^{2} p(x)=p_{2}(y)
$$

follows for all $x, y$ in $G$. On the other hand,

$$
\lim _{\gamma} \frac{1}{2} \Delta_{y}^{2} \varphi_{\gamma}(x)=B(y, y) \lim _{\gamma} c_{\gamma}
$$


holds for all $x, y$ in $G$, hence the $\operatorname{limit}_{\lim } c_{\gamma}=c$ exists and is different from zero, which gives $B(x, x)=\frac{1}{c} p_{2}(x)$ for all $x$ in $G$ and this is impossible.

We infer that any exponential monomial $\varphi$ in $V$ is actually a polynomial of degree at most 1 , which satisfies

$$
\Delta_{y}^{2} \varphi(x)=0
$$

for each $x, y$ in $G$, hence any function in the closed linear hull of the exponential monomials in $V$ satisfies this equation. However $f$ does not satisfy (3.3), hence the linear hull of the exponential monomials in $V$ is not dense in $V$.

Using this theorem we are in the position to disprove the result Theorem 3.1 of Elliot. In what follows $\mathbb{Z}^{\omega}$ denotes the (non-complete) direct sum of countably many copies of the additive group of integers, or, in other words, the set of all finitely supported $\mathbb{Z}$-valued functions on the nonnegative integers.

Theorem 3.4. Spectral synthesis fails to hold on any Abelian group with torsion free rank at least $\omega$.

Proof. First of all we will show that there exists a symmetric bi-additive function $B: \mathbb{Z}^{\omega} \times \mathbb{Z}^{\omega} \rightarrow \mathbb{C}$ with the property that there are infinitely many linearly independent functions of the form $x \mapsto B(x, y)$, where $y$ is in $\mathbb{Z}^{\omega}$. For any nonnegative integer $n$ let $p_{n}$ denote the projection of the direct sum $\mathbb{Z}^{\omega}$ onto the $n$-th copy of $\mathbb{Z}$. This means that for any $x$ in $\mathbb{Z}^{\omega}$ the number $p_{n}(x)$ is the coefficient of the characteristic function of the singleton $\{n\}$ in the unique representation of $x$. It is clear that the functions $p_{n}$ are additive and linearly independent for different choices of $n$. Let

$$
B(x, y)=\sum_{n} p_{n}(x) p_{n}(y)
$$

for each $x, y$ in $\mathbb{Z}^{\omega}$. The sum is finite for any fixed $x, y$, and obviously $B$ is symmetric and bi-additive. On the other hand, if $\chi_{k}$ is the characteristic function of the singleton $\{k\}$, then we have

$$
B\left(x, \chi_{k}\right)=\sum_{n} p_{n}(x) p_{n}\left(\chi_{k}\right)=p_{k}(x),
$$

hence the functions $x \mapsto B\left(x, \chi_{k}\right)$ are linearly independent for different nonnegative integers $k$.

The next step is to show that if $G$ is an Abelian group, $H$ is a subgroup of $G$ and $B: H \times H \rightarrow \mathbb{C}$ is a symmetric, bi-additive function, then $B$ extends to a symmetric bi-additive function on $G \times G$. Then the extension obviously satisfies the property given in Theorem 3.3 and our statement follows. On the other hand, the existence of the desired extension is proved in [7], Theorem 2.

The proof is complete.

By this theorem Lefranc's result is the best possible for free Abelian groups: spectral synthesis holds exactly on the finitely generated ones. Hence the following 
question naturally arises: can spectral synthesis hold on non-finitely generated Abelian groups? If the answer is "yes" then we can ask: is it true that if spectral synthesis fails to hold on an Abelian group, then its torsion free rank is at least $\omega$ ? In the subsequent paragraphs we shall give partial answers to these questions.

\section{Spectral synthesis on Abelian torsion groups}

In [11] we proved the following theorem.

Theorem 4.1. Spectral synthesis holds on any Abelian torsion group.

Proof. Let $V$ be a proper variety in $\mathcal{C}(G)$ and let $W$ denote the linear span of the set of all characters contained in $V$. We have to prove that $W$ is dense in $V$. Supposing the contrary there exists a finitely supported measure $x$ on $G$ such that $\langle x, \gamma\rangle=0$ whenever $\gamma$ is a character in $V$, but $\left\langle x, f_{0}\right\rangle \neq 0$ for some $f_{0}$ in $V$.

Let $J$ denote the support of $x$; then $J$ is a finite subset of $G$. Let $\mathcal{H}$ denote the family of all finite subgroups of $G$ containing $J$. For every $H$ in $\mathcal{H}$ let $V_{H}$ denote the set of the restrictions of the elements of $V$ to $H$. It is easy to check that $V_{H}$ is a variety in $\mathcal{C}(H)$. Whenever a function $\Phi$ is defined on $J$ then we put $\langle x, \Phi\rangle=\sum_{g \in J} x(g) \Phi(g)$. If $H$ is in $\mathcal{H}$ then $\left\langle x, f_{0} \mid H\right\rangle=\left\langle x, f_{0}\right\rangle \neq 0$. Since spectral synthesis holds on $H$ and $f_{0} \mid H$ belongs to $V_{H}$, there is a character $\gamma_{H}$ of $H$ such that $\gamma_{H}$ belongs to $V_{H}$ and $\left\langle x, \gamma_{H}\right\rangle \neq 0$.

Hence we have a net $\left(\gamma_{H}\right)$ along the directed set $\mathcal{H}$ in the product space $\mathbb{T}^{G}$ ( $\mathbb{T}$ is the complex unit circle). From its compactness it follows that this net has an accumulation point, that is, there is a function $\gamma_{0}: G \rightarrow \mathbb{T}$ such that for every finite subset $F$ of $G$ and for every $\varepsilon>0$ there exists an $H$ in $\mathcal{H}$ with $F \cup J$ is included in $H$ and $\left|\gamma_{0}(g)-\gamma_{H}(g)\right|<\varepsilon$ holds for each $g$ in $F$. It is clear that $\gamma_{0}$ is a character of $G$. As $V$ is closed, we also have that $\gamma_{0}$ belongs to $V$.

Since each element $g$ in $J$ has a finite order, the set of values $\gamma(g)$, where $\gamma$ is a character and $g$ is in $J$ is finite. This implies that the set $\left\langle x, \gamma_{H}\right\rangle$ for $H$ in $\mathcal{H}$ is a finite set of complex numbers. As $\left\langle x, \gamma_{0}\right\rangle$ is one of these numbers it follows $\left\langle x, \gamma_{0}\right\rangle \neq 0$. This, however, contradicts the fact that $\gamma_{0}$ is in $V$.

This theorem shows that there are non-finitely generated Abelian groups on which spectral synthesis holds. Hence we can formulate a quite reasonable conjecture: spectral synthesis holds on an Abelian group if and only if its torsion free rank is finite.

\section{Characterization of Abelian groups with spectral analysis and spectral synthesis}

In [12] M. Laczkovich and G. Székelyhidi proved the following result.

Theorem 5.1. Spectral analysis holds on an Abelian group if and only if its torsion free rank is less than the continuum. 
According to Theorem 3.4 there are Abelian groups on which spectral analysis holds and spectral synthesis fails to hold: for instance, any Abelian group with torsion free rank $\omega$, like $\mathbb{Z}^{\omega}$ above. On the other hand, a complete description of those Abelian groups on which spectral synthesis holds is still missing. The conjecture formulated in the previous section has neither been proved nor disproved yet. An interesting situation can be presented by the additive group of rational numbers. It is not known if spectral synthesis holds on this group. Actually, this group is not finitely generated, however, its torsion free rank is 1 . If spectral synthesis does not hold on the rationals, then the above conjecture is drastically disproved: for Abelian groups with torsion free rank zero spectral synthesis holds, as these are exactly the torsion groups. The next simplest case is obviously the case of torsion free rank 1 . On the other hand, if spectral synthesis holds on the rational group, then this is the first example for a torsion free group where spectral synthesis holds and the group is not finitely generated.

In addition to the above conjecture in [13] we proved the following theorem.

Theorem 5.2. The torsion free rank of any Abelian group is equal to the dimension of the linear space consisting of all complex additive functions of the group in the sense that either both are finite and equal, or both are infinite.

Proof. Let $G$ be an Abelian group and let let $k=r_{0}(G) \leqslant+\infty$. Then $G$ has a subgroup isomorphic to $\mathbb{Z}^{k}$. If $k$ is infinite then this is equal to the non-complete direct product of $k$ copies of $\mathbb{Z}$. We will identify this subgroup with $\mathbb{Z}^{k}$. Obviously $\mathbb{Z}^{k}$ has at least $k$ linearly independent complex additive functions; for instance we can take the projections onto the different factors of the product group. On the other hand, by the above mentioned result in [3] any homomorphism of a subgroup of an Abelian group into a divisible Abelian group can be extended to a homomorphism of the whole group. As the additive group of complex numbers is obviously divisible, the above mentioned linearly independent complex additive functions of $\mathbb{Z}^{k}$ can be extended to complex homomorphisms of the whole group $G$, and the extensions are clearly linearly independent, too. Hence the dimension of the linear space of all complex additive functions of $G$ is not less then the torsion free rank of $G$.

Now we suppose that $k<+\infty$. Let $\Phi$ denote the natural homomorphism of $G$ onto the factor group with respect to $\mathbb{Z}^{k}$. As it is a torsion group, hence for each element $g$ of $G$ there is a positive integer $n$ such that

$$
0=n \Phi(g)=\Phi(n g),
$$

thus $n g$ belongs to the kernel of $\Phi$, which is $\mathbb{Z}^{k}$. This means that there exist integers $m_{1}, m_{2}, \ldots, m_{k}$ such that

$$
n g=\left(m_{1}, m_{2}, \ldots, m_{k}\right) .
$$

Suppose now that there are $k+1$ linearly independent complex additive functions $a_{1}, a_{2}, \ldots, a_{k+1}$ on $G$. Then there exist elements $g_{1}, g_{2}, \ldots, g_{k+1}$ in $G$ such that the 
$(k+1) \times(k+1)$ matrix $\left(a_{i}\left(g_{j}\right)\right)$ is regular. For $l=1,2, \ldots, k$ we let $e_{l}$ denote the vector in $\mathbb{C}^{k}$ whose $l$-th coordinate is 1 , the others are 0 . By our above consideration there are integers $m_{l}^{(j)}, n_{j}$ for $l=1,2, \ldots, k$ and $j=1,2, \ldots, k+1$ such that

$$
n_{j} g_{j}=\left(m_{1}^{(j)}, m_{2}^{(j)}, \ldots, m_{k}^{(j)}\right) .
$$

Hence we have

$$
\begin{gathered}
a_{i}\left(n_{j} g_{j}\right)=a_{i}\left(m_{1}^{(j)}, m_{2}^{(j)}, \ldots, m_{k}^{(j)}\right)= \\
=m_{1}^{(j)} a_{i}\left(e_{1}\right)+m_{2}^{(j)} a_{i}\left(e_{2}\right)+\cdots+m_{k}^{(j)} a_{i}\left(e_{k}\right),
\end{gathered}
$$

and therefore

$$
a_{i}\left(g_{j}\right)=\sum_{l=1}^{k} \frac{m_{l}^{(j)}}{n_{j}} a_{i}\left(e_{l}\right)
$$

holds for $i, j=1,2, \ldots, k+1$. This means that the linearly independent columns of the matrix $\left(a_{i}\left(g_{j}\right)\right)$ are linear combinations of the columns of the matrix $\left(a_{i}\left(e_{l}\right)\right)$ for $i=1,2, \ldots, k+1 ; l=1,2, \ldots, k$. But this is impossible, because the latter matrix has only $k$ columns, hence its rank is at most $k$.

We have shown that if the torsion free rank of $G$ is the finite number $k$ then the dimension of the linear space consisting of all complex additive functions of $G$ is at most $k$, hence the theorem is proved.

Another characterization of Abelian groups with finite torsion free rank is given by the following result (see [13]).

Theorem 5.3. The torsion free rank of an Abelian group is finite if and only if any complex bi-additive function is a bilinear function of complex additive functions.

Hence our conjecture has two more equivalent formulations:

- Spectral synthesis holds on an Abelian group if and only if there are only finitely many linearly independent additive functions on the group.

- Spectral synthesis holds on an Abelian group if and only if any complex biadditive function is a bilinear function of complex additive functions.

\section{References}

[1] Schwartz, L., Théorie génerale des fonctions moyenne-périodiques, Ann. of Math., Vol. 48(2) (1947), 857-929.

[2] Lefranc, M., L'analyse harmonique dans $\mathbb{Z}^{n}$, C. R. Acad. Sci. Paris, Vol. 246 (1958), 1951-1953.

[3] Hewitt, E., Ross, K., Abstract Harmonic Analysis I.,II., Springer Verlag, Berlin, 1963.

[4] Elliot, M. J., Two notes on spectral synthesis for discrete Abelian groups, Proc. Cambridge Phil. Soc., Vol. 61 (1965), 617-620. 
[5] Djokovič, D. Z., A representation theorem for $\left(X_{1}-1\right)\left(X_{2}-1\right) \ldots\left(X_{n}-1\right)$ and its applications, Ann. Polon. Math., Vol. 22 (1969), 189-198.

[6] SzÉKelyhidi, L., Convolution type functional equations on topological Abelian groups, World Scientific Publishing Co. Pte. Ltd., Singapore, New Jersey, London, Hong Kong, 1991.

[7] SzéKelyhidi, L., On the extension of exponential polynomials, Math. Bohemica, vol. 125 , no. 3, pp. 365-370, 2000.

[8] Székelyhidi, G., Spectral Synthesis on Locally Compact Abelian Groups, (essay), Cambridge, Trinity College, 2001.

[9] SzÉkelyhidi, L., A Wiener Tauberian theorem on discrete abelian torsion groups, Annales Acad. Paedag. Cracov., Studia Mathematica I., Vol. 4 (2001), 147-150.

[10] SzÉKelyhidi, L., The failure of spectral synthesis on some types of discrete Abelian groups, Jour. Math. Anal. Appl., Vol. 291 (2004), 757-763.

[11] Bereczky, A., Székelyhidi, L., Spectral synthesis on torsion groups, Jour. Math. Anal. Appl., Vol. 304/2, (2005) 607-613.

[12] Laczkovich, M., Székelyhidi, G., Harmonic analysis on discrete Abelian groups, to appear in Proc. Amer. Math. Soc.

[13] SzÉkelyhidi, L., Polynomial functions and spectral synthesis, to appear in Aequationes Math.

\section{László Székelyhidi}

Institute of Mathematics

University of Debrecen

4010 Debrecen, Pf. 12. Hungary 